\title{
BMJ Open Road to recovery: a study protocol quantifying neurological outcome in cardiac surgery patients and the role of cerebral oximetry
}

\author{
Joanna S Semrau (D) , ${ }^{1}$ Stephen H Scott, ${ }^{1,2}$ Andrew G Hamilton, ${ }^{3,4}$ \\ Dimitri Petsikas,, Darrin M Payne, ${ }^{3,4}$ Gianluigi Bisleri,, ${ }^{3,4}$ Tarit Saha, 5,6 \\ John Gordon Boyd ${ }^{7}$
}

To cite: Semrau JS, Scott SH, Hamilton AG, et al. Road to recovery: a study protocol quantifying neurological outcome in cardiac surgery patients and the role of cerebral oximetry. BMJ Open 2019;9:e032935. doi:10.1136/ bmjopen-2019-032935

- Prepublication history for this paper is available online. To view these files, please visit the journal online (http://dx.doi org/10.1136/bmjopen-2019032935).

Received 12 July 2019 Revised 23 September 2019 Accepted 17 October 2019
Check for updates

(C) Author(s) (or their employer(s)) 2019. Re-use permitted under CC BY-NC. No commercial re-use. See rights and permissions. Published by BMJ.

For numbered affiliations see end of article.

Correspondence to Dr John Gordon Boyd; gordon.boyd@kingstonhsc.ca

\section{ABSTRACT}

Introduction Patients undergoing cardiac surgery may experience both short-term and long-term postoperative neurological problems. However, the underlying cause of this impairment is unclear. Regional cerebral oxygen saturation $\left(\mathrm{rSO}_{2}\right.$ ) levels may play a role in the development of acute dysfunction, known as postoperative delirium, in addition to longer term outcomes after cardiac surgery. Yet the degree of impairment has been difficult to define, partly due to subjective methods of assessments. This study aims to fill this knowledge gap by determining the relationship between $\mathrm{rSO}_{2}$, postoperative delirium and long-term neurological outcome after cardiac surgery using quantitative robotic technology.

Methods and analysis 95 patients scheduled for elective cardiac surgery will be recruited for this single-centre prospective observational study. Patients will be assessed before as well as 3 and 12 months after their surgery using the Kinarm End-Point Lab and standardised tasks. Intraoperatively, $\mathrm{rSO}_{2}$ and other haemodynamic data will be collected for the duration of the procedure. Following their operation, patients will also be screened daily for delirium during their hospital stay.

Ethics and dissemination This study has been approved by the Health Sciences Research Ethics Board at Queen's University (DMED-1672-14). The results of this study will be published in a peer-review journal and presented at international and/or national conferences as poster or oral presentations. Participants wishing to know the results of this study will be contacted directly on data publication. Trial registration number NCT04081649

\section{INTRODUCTION}

Postoperative neurological dysfunction following cardiac surgery can be a devastating outcome for the patient and their family. Deficits may begin to appear early in the form of postoperative delirium, which has been observed to occur in $10 \%$ to $30 \%$ of cardiac surgery patients. ${ }^{12}$ These patients may be at risk for developing future complications and may require more at home and/ or nursing home care. ${ }^{3}$ In particular, patients

\section{Strengths and limitations of this study}

- This is a properly powered single-centre study that includes a statistical plan with both primary and secondary analyses.

- While our study provides a novel and quantitative approach to neurological assessment, no statistical comparisons between quantified and pen-andpaper assessments will be performed due to the varying nature of these tests.

- This will be the first study that will include both delirium and cerebral oxygen saturation as predictors of quantified long-term neurological function.

who present with delirium following cardiac surgery may also be at risk for developing long-term cognitive dysfunction. ${ }^{4}$

Neurological dysfunction following cardiac surgery can persist for months following physical recovery. Postoperative cognitive impairment has been reported to occur in about $20 \%$ to $40 \%$ of patients weeks to months after their procedure. ${ }^{5}$ The cause, however, is most likely multifactorial. Prior stroke, diabetes, age and severity of cardiovascular disease have all been shown to contribute to poor neurological function after cardiac surgery. ${ }^{6}$ Certain procedures may also be associated with greater risks of neurological dysfunction. Research groups have shown that patients after valve replacement surgery have significantly worse cognitive functioning than patients following coronary artery bypass grafting (CABG) surgery. ${ }^{78}$ Combined surgeries, often involving longer bypass times, may also increase the risk of delirium. ${ }^{9}$ Procedures requiring entry into the heart involve increased embolic load, generating the possibility of postoperative neurological impairment. ${ }^{10}$ 
Intraoperative regional cerebral oxygen saturation $\left(\mathrm{rSO}_{2}\right)$, measured by near-infrared spectroscopy, may also play a role in postoperative neurological functioning. Prolonged desaturations in the brain have been associated with poor neurological outcome, as well as delirium, and active monitoring to prevent significant drops in $\mathrm{rSO}_{2}$ may prevent these complications. ${ }^{11}{ }^{12}$ Intraoperative $\mathrm{rSO}_{2}$ monitoring has gradually become regular practice in many hospitals, yet the supporting evidence is lacking in quality. ${ }^{13}{ }^{14}$ A review by $\mathrm{Yu}$ and colleagues concluded that the role of $\mathrm{rSO}_{2}$ monitoring in postoperative neurological outcome is uncertain, and more research needs to be performed to fully understand this relationship. ${ }^{14}$ These inconsistent findings may be due to the subjective nature of neurological assessments used in these studies. Standard neuropsychological testing can also vary in comprehensiveness, definitions of impairment and often do not include measures of sensory or motor function. ${ }^{1315}$

Robotic technology may offer a solution to this problem by providing a quantitative approach to neurological assessment. The Kinarm robotic platform (Kinarm, Kingston, Canada) provides an objective approach to assess upper limb sensory, motor and cognitive function. The Kinarm has been previously used to quantify impairment in patients following stroke and detected deficits which were not apparent in routine clinical testing. ${ }^{16}$ The Kinarm tasks have also been able to generate a detailed neurological phenotype for transient ischaemic attack and traumatic brain injury. ${ }^{17} 18$ Our early work recently established the feasibility of using the Kinarm as a novel perioperative assessment method in CABG surgery patients. ${ }^{19}$

The overall goal of this research programme is to determine quantitative predictors of quantitative longterm neurological functioning following different cardiac surgery procedures. We aim to generate a timeline of neurological function that includes pre-existing performance, postoperative delirium and neurological outcome. Furthermore, we will test the hypothesis that intraoperative $\mathrm{rSO}_{2}$ desaturations, incidence of postoperative delirium and pre-existing impairment can predict neurological dysfunction following cardiac surgery.

\section{METHODS AND ANALYSIS}

\section{Patient involvement}

Adult patients (aged 18 years or older) undergoing cardiac surgery (CABG, valve or combined) will be recruited for the Cerebral Oxygenation and Neurological FUnctioning after cardiac SurgEry (CONFUSE) study at Kingston Health Sciences. Patients with a history of neurodegenerative disorder or diagnosed impairment will be excluded. Recruited patients will not be actively involved in determining the design, recruitment or outcome measures for this study.

This study has been approved by the Health Sciences Research Ethics Board at Queen's University. The study timeline for the CONFUSE study is illustrated in figure 1.

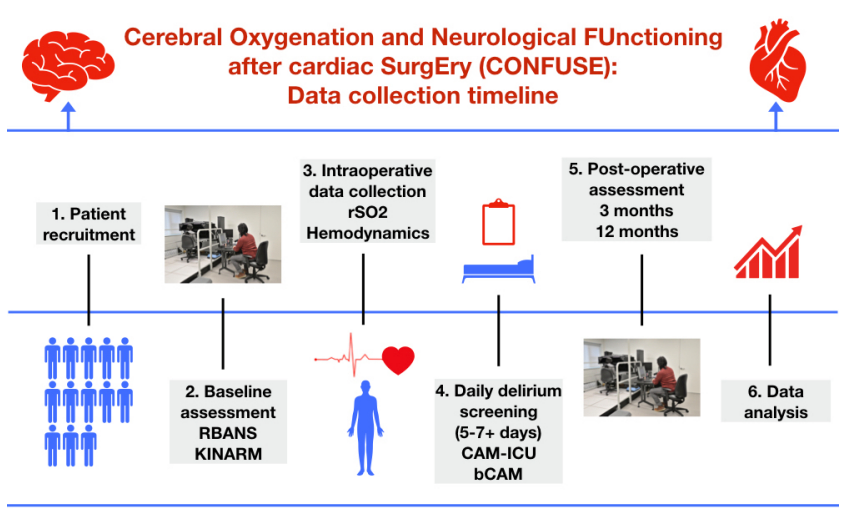

Figure 1 Data collection timeline for the CONFUSE study. bCAM, brief confusion assessment method;CAM-ICU, Confusion Assessment Method for the ICU; CONFUSE, Cerebral Oxygenation and Neurological FUnctioning after cardiac SurgEry; ICU, intensive care unit; RBANS, Repeatable Battery for the Assessment of Neuropsychological Status; $\mathrm{rSO}_{2}$, regional cerebral oxygen saturation.

Recruitment for patients undergoing aortic valve replacement started in April 2018. We initially focused our recruitment on this group to achieve greater homogeneity in our sample. However, due to low recruitment rates (about one patient every 2 months), inclusion criteria was altered to include a variety of different cardiac procedures. Due to the expected increase of heterogeneity in this study, our statistical analysis will account for any possible group differences.

\section{Intraoperative data collection}

During surgery, $\mathrm{rSO}_{2}$ will be collected using the FORESIGHT cerebral oximeter (CAS medical systems, Branford, CT, USA) following the same protocol as previous studies. ${ }^{20}$ An adult sensor will be placed on the middle of each patient's forehead to detect the ratio of deoxyhaemoglobin/oxyhaemoglobin in the vascular bed of the frontal cortex. Cerebral oxygen data will be recorded every $2 \mathrm{~s}$ for the duration of the procedure. Haemodynamic data (systolic/diastolic blood pressure, heart rate, central venous pressure, etc) will also be collected intraoperatively at $2 \mathrm{~s}$ intervals.

\section{Postoperative delirium screening}

Patients will be screened for delirium in the first 24 hours using the Confusion Assessment Method for the intensive care unit (CAM-ICU) during their stay in the cardiac ICU. Following transfer to the floor, patients will be screened daily for delirium using the brief confusion assessment method (bCAM) for the duration of their stay at the hospital. The bCAM and CAM-ICU were developed from the same algorithm to detect delirium on the floor and the ICU, respectively. ${ }^{21}$ Both assessment methods assess the four main features of delirium: altered mental status or fluctuating course, inattention, altered level of consciousness and disorganised thinking. 


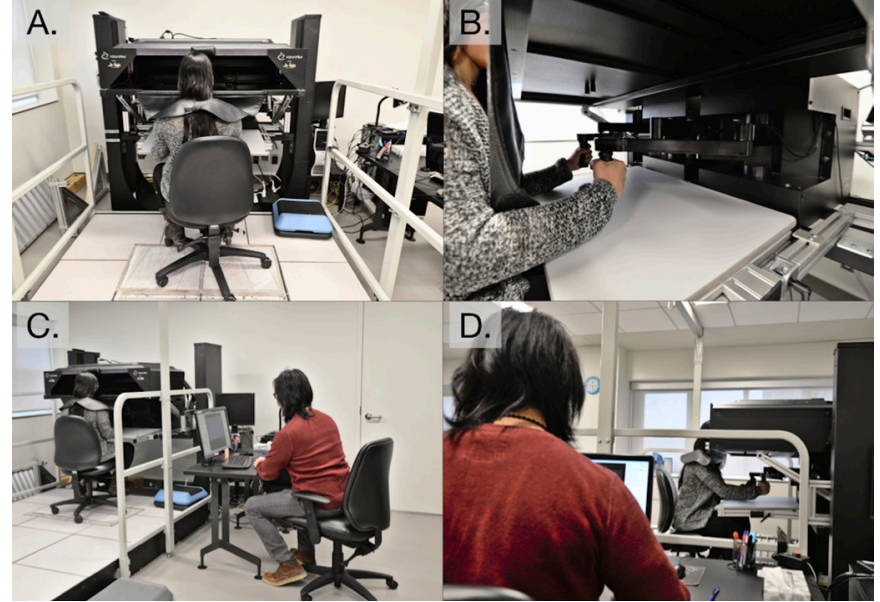

Figure 2 Photos of the Kinarm robotic system set-up. (A) Patient will be seated in front of the robot so that they are able to view the horizontal screen. (B) Using the robot's handles, patients will complete eight standardised tasks measuring cognitive, sensory and motor function. (C) and (D) A trained research associate will lead the assessment session. Photos are used with permission.

\section{Neurological assessment}

Patients will undergo comprehensive neurological assessment prior to surgery, as well as 3 and 12 months postoperation. A trained research associate will perform the assessment. Patients will be assessed using the Kinarm End-Point Lab (Kinarm, Kingston, Canada), and the Repeatable Battery for the Assessment of Neuropsychological Status (RBANS). The RBANS is a standardised pen-and-paper questionnaire used to detect cognitive decline. ${ }^{22}$ Five major cognitive domains are assessed using the RBANS: immediate memory, visuospatial construction, attention, language and delayed memory. The total scale is determined by summarising all five domain scores.

The robotic design and assessment method has been described previously. ${ }^{19}$ To perform the tasks, patients will be seated in front of the robot positioned to grasp the handles that permit movement in the horizontal plane. Participants will have no view of their hand and arm position; all visual feedback will be through the robot's virtual reality system aligned with the horizontal workspace (figure 2). Each patient will perform eight standardised tasks, assessing sensory, motor and cognitive functioning (figure 3). Each task is associated with six to twelve parameters (reaction time, posture speed, initial movement angle, etc) that quantify the degree of neurological function. Parameters are adjusted for age, sex and handedness then summarised into an overall task score. Task scores are represented as one-sided z-like scores, with 0 denoting best performance and any score above 1.96 denoting performance worse than $95 \%$ of healthy controls (here defined as impaired). The robotic tasks are described below.

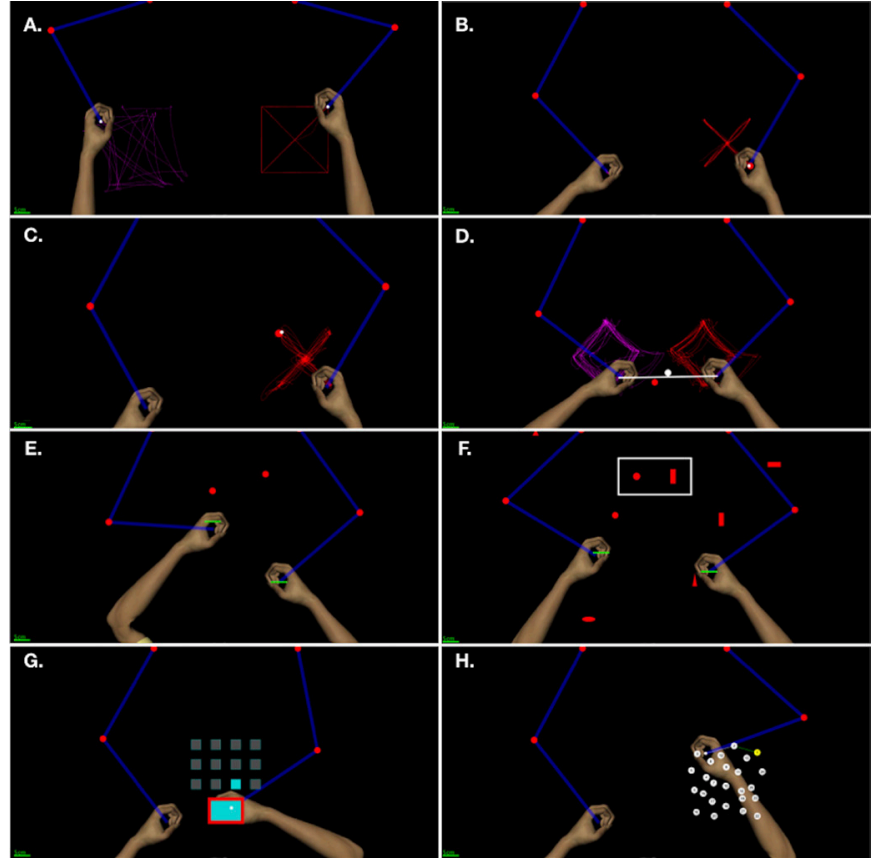

Figure 3 Kinarm standardised tasks for neurological assessment. (A) Arm position matching. (B) Visually-guided reaching. (C) Reverse visually-guided reaching. (D) Ball on bar. (E) Object hit. (F) Object hit and avoid. (G) Spatial span. (H) Trail making test. Arms and hand paths shown in purple (left hand) and red (right hand) are for illustrative purposes only and are not visible to the participant.

\section{Kinarm tasks}

\section{Arm position matching}

Patients are instructed to mirror-match the movement and final position of the robot-controlled arm. This task is performed twice to obtain data on both arms. Patients will require intact proprioceptive functioning to process information on where their limb is in the horizontal space. This arm position matching task has been shown to determine the degree of position sense impairment in subjects following stroke (figure $3 \mathrm{~A}$ ). ${ }^{23}$

\section{Visually-guided reaching}

This centre-out reaching task assesses basic visuospatial skills and sensorimotor control. ${ }^{16}$ The subject's hand is represented by a white dot. Each subject is then instructed to bring the white dot quickly and accurately to the red target as they appear on the screen (figure 3B).

\section{Reverse visually-guided reaching}

This task is presented similarly to the visual-guided reaching task. However, the movement of the white dot is now reversed compared with the movement of the patient's hand. To successfully complete the task, patients must move their hand away from the target, thereby reaching the red target with the white dot. This is a more complex reaching task, requiring inhibitory control to not automatically reach towards the red target and a cognitive rule to move the white cursor by moving in the opposite direction. Visuomotor impairments of this nature have 
been described in individuals at risk of developing Alzheimer's disease (figure 3C). ${ }^{24}$

\section{Ball on bar}

Patients are instructed to use a virtual bar, generated between their hands, to balance a virtual ball as it appears on the screen. The subject must then bring the balanced ball on bar to different target locations in virtual space. This task consists of three levels: (1) ball fixed to bar, (2) ball moves along bar based on bar orientation, (3) ball can roll freely along bar. This task assesses bimanual motor control (figure 3D). ${ }^{25}$

\section{Object hit}

In this task, the participants' hands are represented by paddles. Subjects are instructed to hit balls falling down from the top of the screen towards them. As the task progresses, the balls begin to fall more frequently and increase in speed, making the task gradually more difficult. This simple task measures sensorimotor function and decision-making (figure $3 \mathrm{E}$ ). ${ }^{26}$

\section{Object hit and avoid}

This task is similar to the object hit task, where patients use the robot's handles to hit objects falling from the top of the screen towards them. In this task however, patients are instructed to only hit two target shapes while avoiding all other shapes (ie, distractors). The object hit and avoid task therefore requires additional cognitive functions related to attention to identify each shape and inhibitory control to avoid the distractors (figure $3 \mathrm{~F}$ ). ${ }^{27}$

\section{Spatial span}

A series of squares light up on the screen in a particular sequence. Within a 3-by-4 grid of squares, patients are required to replay the sequence by reaching with their dominant hand to the correct squares. This task assesses visuospatial working memory, and is similar to the Corsi block-tapping task (figure 3G). ${ }^{28}$

\section{Trail making}

This task is a quantitative version of the standard penand-paper neuropsychological test. ${ }^{29}$ In trailing making part A, patients are instructed to connect the numbers 1 through 25. The trail making part B task requires subjects to connect the dots by alternating numbers and letters (1-A-2-B-3-C, etc). This task assesses executive function related to task switching (figure $3 \mathrm{H}$ ).

\section{Statistical analysis}

\section{Primary outcome analysis}

Our primary outcome measure is postoperative neurological functioning as measured by the Kinarm (ie, global performance scores for each Kinarm task). Multiple linear regression modelling will be used to determine how much variance in postoperative neurological functioning is explained by our main predictor, $\mathrm{rSO}_{2}$. We assume that 12 month performance will correlate with their 3 month performance for each patient, reducing our analysis to one outcome. To confirm our assumption, two sets of models will be performed using 3 and 12 month scores as our outcome. Provided that both sets of models indicate similar predictors, our primary outcome will be postoperative neurological functioning 12 months after surgery. Any intraindividual differences between preoperative, 3 month and 12 month postoperative functioning will be explored in our secondary analyses.

Measurements of $\mathrm{rSO}_{2}$ used in our primary analysis will be mean $\mathrm{rSO}_{2}$, and time spent below baseline $\mathrm{rSO}_{2}$, measured as an area under the curve (AUC). Baseline $\mathrm{rSO}_{2}$ will be determined in the first 5 min prior to induction of anaesthesia. Our earlier work demonstrated that subtle and undiagnosed impairments prior to surgery detected by the Kinarm can highly predict postoperative neurological dysfunction. ${ }^{30}$ This and other studies have strongly emphasised the importance of including baseline functioning, as it can account for a significant amount of variance in neurological outcome following cardiac surgery. ${ }^{4} 1$ Moving forward, we aim to control for preoperative functioning, and include it as a covariate in our model. Furthermore, we will include delirium as an additional predictor of postoperative neurological functioning. We will perform separate models for each Kinarm assessment, for a total of seven models. Corrections will be made for multiple comparisons. For a model with four predictors (preoperative task score, postoperative delirium, mean $\mathrm{rSO} 2$ and AUC rSO2), and one outcome (postoperative task score), and assessing for a small effect size (Cohen's $d=0.15$ ), a sample size of 95 will be required to achieve $85 \%$ power at alpha $=0.05$. All statistical analysis and figure generation will be performed using $\mathrm{R}$ software. ${ }^{32}$ Power analysis was performed using G Power. ${ }^{33}$

\section{Secondary outcome analysis — Intraindividual changes in neurological dysfunction}

As a secondary method of analysing perioperative neurological performance, we will use multilevel modelling for repeated measures with three time points: preoperative performance, 3 month and 12 month postoperative performance. Incidence of delirium and $\mathrm{rSO}_{2}$ measures will be additional predictors for this model. In contrast to linear regression, multilevel modelling allows us to generate a longitudinal growth model, illustrating the trajectory of each patient's recovery process. In addition, multilevel modelling allows for missing values, allowing data to be used when patients are lost to follow-up. This analysis method will highlight any variability occurring between or within patients; effects that may have been span style='font-family: 'Times New Roman">mitigated in our primary model. This research programme may not be powered to detect a meaningful effect using this multilevel model approach and will therefore be exploratory. 
Secondary outcome analysis - Intraoperative haemodynamic data

Preliminary correlational analysis will be performed between $\mathrm{rSO}_{2}$ and other haemodynamic variables (arterial blood pressure, heart rate, temperature, peripheral oxygen saturation and blood gases) will be performed using Spearman's rho coefficients. An in-depth examination of intraoperative variables will involve time series analysis. In particular, we are interested in determining predictors of $\mathrm{rSO}_{2}$ over time as a measure of patients' cerebral autoregulation. A study performed by Lee $e t$ al. determined that time-varying cerebral autoregulation has been shown to correlate with the duration of delirium among critically ill patients. ${ }^{34}$ This approach will allow us to detect trends in the relationship between these variables over time, and explore how these trends predict delirium and postoperative neurological functioning.

\section{ETHICS AND DISSEMINATION}

Patients are recruited during the presurgical screening appointment by a research associate/research nurse. This research protocol does not interfere with standard of care at the Kingston Health Sciences Centre. Patients are carefully monitored during assessment times for any signs of discomfort, and assessment can be stopped at any time by the trained research associate. Confidential information will be safely stored.

Following data collection and analysis, results of the study will be published in a peer-reviewed journal. Data will also be presented at international and national conferences. Study participants who expressed interest in receiving the results of this study will be contacted directly and sent results in the form of publications, abstracts and/or poster presentations.

\section{Author affiliations \\ ${ }^{1}$ Centre for Neuroscience Studies, Queen's University, Kingston, Ontario, Canada \\ ${ }^{2}$ Biomedical and Molecular Sciences, Queen's University, Kingston, Ontario, Canada \\ ${ }^{3}$ Cardiac Surgery, Kingston General Hospital, Kingston, Ontario, Canada \\ ${ }^{4}$ Surgery, Queen's University, Kingston, Ontario, Canada \\ ${ }^{5}$ Anesthesiology and Perioperative Medicine, Kingston General Hospital, Kingston, Ontario, Canada \\ ${ }^{6}$ Anesthesiology and Perioperative Medicine, Queen's University, Kingston, Ontario, Canada}

${ }^{7}$ Critical Care Medicine, Kingston General Hospital, Kingston, Ontario, Canada

\section{Twitter Joanna S Semrau @jssemrau}

Contributors JSS prepared the manuscript, designed the statistical plan and performed sample size calculations. AGH, DP, DMP, GB, TS and SHS constructively contributed to the manuscript and the protocol plan. JGB conceptualised the project and is the acting supervisor of this study. All authors have read and approved this manuscript prior to submission.

Funding This work was supported by the Southeastern Ontario Academic Medical Organization (SEAM0) Innovation fund.

Competing interests JSS, AGH, DMP, DP, GB and TS have no competing interests. SHS is the inventor of the KINARM robot and co-founder of BKIN technologies. JGB receives a stipend from the Trillium of Life Network for his role as a hospital donation physician.

Patient consent for publication Not required.
Provenance and peer review Not commissioned; externally peer reviewed.

Open access This is an open access article distributed in accordance with the Creative Commons Attribution Non Commercial (CC BY-NC 4.0) license, which permits others to distribute, remix, adapt, build upon this work non-commercially, and license their derivative works on different terms, provided the original work is properly cited, appropriate credit is given, any changes made indicated, and the use is non-commercial. See: http://creativecommons.org/licenses/by-nc/4.0/.

ORCID iD

Joanna S Semrau http://orcid.org/0000-0003-3529-2499

\section{REFERENCES}

1 Järvelä K, Porkkala H, Karlsson S, et al. Postoperative delirium in cardiac surgery patients. J Cardiothorac Vasc Anesth 2018;32:1597-602.

2 Bakker RC, Osse RJ, Tulen JHM, et al. Preoperative and operative predictors of delirium after cardiac surgery in elderly patients. Eur $J$ Cardiothorac Surg 2012;41:544-9.

3 Mangusan RF, Hooper V, Denslow SA, et al. Outcomes associated with postoperative delirium after cardiac surgery. Am J Crit Care 2015;24:156-63.

4 Lingehall HC, Smulter NS, Lindahl E, et al. Preoperative cognitive performance and postoperative delirium are independently associated with future dementia in older people who have undergone cardiac surgery: a longitudinal cohort study. Crit Care Med 2017;45:1295-303.

5 Newman MF, Kirchner JL, Phillips-Bute B, et al. Longitudinal assessment of neurocognitive function after coronary-artery bypass surgery. N Engl J Med 2001;344:395-402.

6 Pérez-Belmonte LM, San Román-Terán CM, Jiménez-Navarro M, et al. Assessment of long-term cognitive impairment after off-pump coronary-artery bypass grafting and related risk factors. J Am Med Dir Assoc 2015;16:263.e9-263.e11.

7 Zimpfer D, Czerny M, Kilo J, et al. Cognitive deficit after aortic valve replacement. Ann Thorac Surg 2002;74:407-12.

8 Hudetz JA, lqbal Z, Gandhi SD, et al. Postoperative delirium and short-term cognitive dysfunction occur more frequently in patients undergoing valve surgery with or without coronary artery bypass graft surgery compared with coronary artery bypass graft surgery alone: results of a pilot study. J Cardiothorac Vasc Anesth 2011;25:811-6.

9 O'Neal JB, Billings FT, Liu X, et al. Risk factors for delirium after cardiac surgery: a historical cohort study outlining the influence of cardiopulmonary bypass. Can J Anaesth 2017;64:1129-37.

10 Patel N, Minhas JS, Chung EML. Intraoperative embolization and cognitive decline after cardiac surgery: a systematic review. Semin Cardiothorac Vasc Anesth 2016;20:225-31.

11 Slater JP, Guarino T, Stack J, et al. Cerebral oxygen desaturation predicts cognitive decline and longer hospital stay after cardiac surgery. Ann Thorac Surg 2009;87:36-45.

12 Momeni M, Meyer S, Docquier M-A, et al. Predicting postoperative delirium and postoperative cognitive decline with combined intraoperative electroencephalogram monitoring and cerebral nearinfrared spectroscopy in patients undergoing cardiac interventions. J Clin Monit Comput 2019;33:999-1009.

13 Zheng F, Sheinberg R, Yee M-S, et al. Cerebral near-infrared spectroscopy monitoring and neurologic outcomes in adult cardiac surgery patients: a systematic review. Anesth Analg 2013;116:663-76.

14 Yu Y, Zhang K, Zhang L, et al. Cerebral near-infrared spectroscopy (NIRS) for perioperative monitoring of brain oxygenation in children and adults. Cochrane Database Syst Rev 2018;1.

15 Rudolph JL, Schreiber KA, Culley DJ, et al. Measurement of postoperative cognitive dysfunction after cardiac surgery: a systematic review. Acta Anaesthesiol Scand 2010;54:663-77.

16 Coderre AM, Zeid AA, Dukelow SP, et al. Assessment of upper-limb sensorimotor function of subacute stroke patients using visually guided reaching. Neurorehabil Neural Repair 2010;24:528-41.

17 Simmatis L, Krett J, Scott SH, et al. Robotic exoskeleton assessment of transient ischemic attack. PLoS One 2017;12:e0188786-13.

18 Debert CT, Herter TM, Scott SH, et al. Robotic assessment of sensorimotor deficits after traumatic brain injury. J Neurol Phys Ther 2012;36:58-67.

19 Semrau JS, Scott SH, Hamilton AG, et al. The relationship between cerebral oxygen saturation and quantitative metrics of neurological function after coronary bypass surgery: a feasibility study. $J$ Cardiovasc Surg 2018;59:716-28.

20 Wood MD, Maslove DM, Muscedere JG, et al. Low brain tissue oxygenation contributes to the development of delirium in 
critically ill patients: a prospective observational study. J Crit Care 2017;41:289-95.

21 Inouye SK, van DCH, Alessi CA. Clarifying confusion: the confusion assessment method. Ann Intern Med 1990;113:941-8.

22 Randolph C, Tierney MC, Mohr E, et al. The repeatable battery for the assessment of neuropsychological status (RBANS): preliminary clinical validity. J Clin Exp Neuropsychol 1998;20:310-9.

23 Dukelow SP, Herter TM, Moore KD, et al. Quantitative assessment of limb position sense following stroke. Neurorehabil Neural Repair 2010;24:178-87.

24 Hawkins KM, Sergio LE. Visuomotor impairments in older adults at increased Alzheimer's disease risk. J Alzheimers Dis 2014;42:607-21.

25 Lowrey CR, Jackson CP, Bagg SD, et al. A novel robotic task for assessing impairments in bimanual coordination post-stroke. Int $J$ Phys Med Rehabil 2014:S3:002.

26 Tyryshkin K, Coderre AM, Glasgow Jl, et al. A robotic object hitting task to quantify sensorimotor impairments in participants with stroke. J Neuroeng Rehabil 2014;11:47.

27 Bourke TC, Lowrey CR, Dukelow SP, et al. A robot-based behavioural task to quantify impairments in rapid motor decisions and actions after stroke. J Neuroeng Rehabil 2016;13:91.
28 Berch DB, Krikorian R, Huha EM. The Corsi block-tapping task: methodological and theoretical considerations. Brain Cogn 1998;38:317-38.

29 Sánchez-Cubillo I, Periáñez JA, Adrover-Roig D, et al. Construct validity of the TRAIL making test: role of task-switching, working memory, inhibition/interference control, and visuomotor abilities. J Int Neuropsychol Soc 2009;15:438-50.

30 Semrau JS, Scott SH, Hamilton AG, et al. Quantified pre-operative neurological dysfunction predicts outcome after coronary artery bypass surgery. Aging Clin Exp Res 2019;0123456789. doi:10.1007/ s40520-019-01184-9. [Epub ahead of print: 08 Apr 2019].

31 Millar K, Asbury AJ, Murray GD. Pre-Existing cognitive impairment as a factor influencing outcome after cardiac surgery. Br $\mathrm{J}$ Anaesth 2001;86:63-7.

32 R Core Team. R: a language and environment for statistical computing, 2016.

33 Faul F, Erdfelder E, Lang A-G, et al. G*Power 3: a flexible statistical power analysis program for the social, behavioral, and biomedical sciences. Behav Res Methods 2007;39:175-91.

34 Lee KF, Wood MD, Maslove DM, et al. Dysfunctional cerebral autoregulation is associated with delirium in critically ill adults. $J$ Cereb Blood Flow Metab 2018;0:1-9. 